\title{
Akute Rhinosinusitis
}

\section{Schneller fit mit Fünf-Pflanzenkombination}

- Die akute Rhinosinusitis (ARS) ist eine sehr häufige Erkrankung. Einer Erhebung in Belgien zufolge machte etwa ein Drittel der Befragten im zurückliegenden Jahr mindestens eine Episode durch. Rund 24\% von ihnen waren deshalb mit Antibiotika behandelt worden. „Das ist eindeutig zuviel", warnte Prof. Claus Bachert, Chefarzt im Fachbereich HNO-Heilkunde am Universitätsklinikum Gent/Belgien. Bei den meisten Patienten sei die Infektion viral bedingt und werde nur in 0,2-2\% der Fälle durch eine bakterielle Superinfektion kompliziert.

\section{Kürzere Leidenszeit}

Eine placebokontrollierte, randomisierte, doppelblinde Multicenterstudie lieferte nun erstmals gute Evidenz für die ARS-Therapie mit einem Phytotherapeutikum [Jund R et al. Rhinology 2012;50:417]. Für die Studie wurden 386 Patienten rekrutiert und von 380 die Daten evaluiert. Die Patienten wurden 15 Tage lang entweder mit dem hoch konzentrierten Trockenextrakt aus
Schlüsselblumenblüten, gelbem Enzian, Eisenkraut, Ampferkraut und Schwarzem Holunder (Sinupret ${ }^{\oplus}$ extract) oder mit Placebo behandelt. Primärer Endpunkt war der Hauptsymptomscore (MSS = major symptom score) bei Therapieende, mit den fünf Symptomen Rhinorrhö, postnasaler Drip, verstopfte Nase, Kopf- und Gesichtsschmerz. Wie Bachert ausführte, fühlten sich die Teilnehmer der Verumgruppe schneller besser. Vom Tag 3 an trennten sich die MSS-Kurven und zeigten eine deutliche Überlegenheit des Pflanzenextrakts. Im Durchschnitt verkürzte sich die Genesungszeit unter Verum um 3,8 Tage.

\section{Multi-Target-Ansatz}

Das Phytopräparat setzt nach Aussage von Prof. André Gessner, Mikrobiologe am Universitätsklinikum Regensburg, an verschiedenen Targets der ARS-Pathophysiologie an. In pharmakologischen Untersuchungen zeigten sich antientzündliche, sekretolytische, antivirale und antibakterielle Wirkungen.

\section{Zusatznutzen durch DPP-4-Hemmer Neuer Goldstandard für Diabetes?}

— Im März 2007 wurde das orale Diabetikum Sitagliptin (Xelevia ${ }^{\circledR}$ ) erstmals in der EU zugelassen. Durch Hemmung des Enzyms Dipeptidylpeptidase-4 wird der Abbau des Hormons GLP-1 verhindert, das die Freisetzung von Insulin stimuliert. Studien über GLP-1 haben Prof. Wolfgang Schmidt, Direktor der Medizinischen Klinik I an der Ruhr-Universität Bochum, zufolge keine unerwünschten Wirkungen zutage gefördert: „Für uns Forscher war es selbst erstaunlich, dass nichts fassbar war." Keine Übelkeit, "kein erhöhtes Risiko" für eine Pankreatitis. Es habe zwar anfangs Verdachtsfälle schwerer Pankreatitis gegeben, diese hätten sich in mehreren Großstudien jedoch nicht erhärtet.

Größter Vorteil von GLP-1: Es bestehe keine Hypoglykämiegefahr. Schmidt ver- wies auf die Studie von Nauck [Nauck et al. Diabetologia 1993;36:741-744], bei der eine GLP-Infusion den Blutzucker normalisierte, die Beta-Zellen stimulierte, allerdings nur bis der Normalzucker erreicht wurde. Dadurch besteht keine Hypoglykämiegefahr. „Dieses Thema wird bisher grob vernachlässigt. Dabei haben wir eine knallharte Datenlage: Schwere Hypoglykämien sind um jeden Preis zu vermeiden", so Schmidt.

\section{Vorteile bei Niereninsuffizienz}

Von Vorteil seien DPP-4-Hemmer auch bei Niereninsuffienz. "Metformin bleibt der Goldstandard bei Preis-Leistung. Aber wo eine Niereninsuffienz besteht, belegen Studien keine Unterlegenheit gegen Metformin", sagte Schmidt.
- Dr. Martina-Jasmin Utzt

Quelle: Symposium „Neue Daten zu einer evidenzbasierten Phytotherapie der akuten Rhinosinusitis"; Nürnberg, Mai 2013 (Veranstalter: Bionorica)

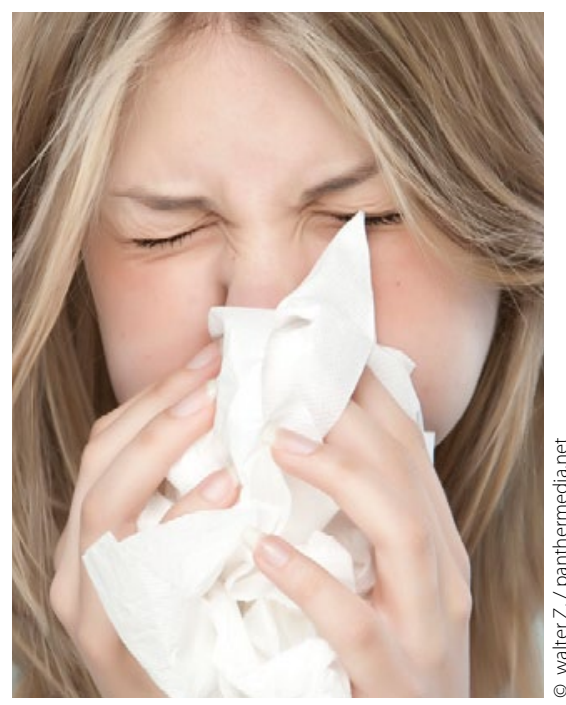

Schnupfen ist lästig. Ihn schnell wieder Isozuwerden - das wünschen sich viele
Wichtig sei insbesondere eine frühe glykämische Kontrolle. „Nach zwölf bis 15 Jahren bringt es nicht mehr viel", betonte Schmidt mit Blick auf eine mögliche Reduktion der kardiovaskulären Mortalität.,,Unsere Crux ist, dass Typ-2-Diabetiker schon vor der Entdeckung eine gewisse Schadenslast mit sich tragen", sagte Andreas Lueg, in Hameln niedergelassener Diabetologe. Er sehe in der Praxis einen Zusatznutzen der DPP-4-Hemmer. "Man kommt an den Vorteilen nicht vorbei", so Lueg, vor allem wenn man die Heterogenität der Patienten berücksichtige.

\footnotetext{
- Robert Büssow

Quelle: Pressekonferenz ${ }_{\text {, }}$ Jahre Xelevia und Velmetia: Eine Bestandsaufnahmen gestern - heute - morgen", 48. Jahrestagung der Deutschen Diabetes Gesellschaft (DDG), Leipzig, Mai 2013 (Veranstalter: Berlin-Chemie)
} 